\title{
Using COVID-19 Narratives to Think Deeply About Physicians' Conflicting Roles and Responsibilities
}

\author{
Jesus Olivera', Richard Gunderman² \\ ${ }^{1}$ Indiana University School of Medicine, ${ }^{2}$ Indiana University School of Medicine, Department of \\ Radiology \& Imaging Sciences, Department of Pediatrics, Department of Medical Education; \\ Indiana University-Purdue University Indianapolis, Department of Medical Humanities and \\ Health Studies, Department of Philosophy
}

Background: In recent decades, many physicians have abandoned the private practice model of medicine, electing instead to work as hospital employees. For the most part, hospitals and physician employees enjoy a synergistic relationship. In other cases, conflicts can arise between the two parties. This paper explores a subset of such conflicts, those which transpired within the context of the COVID-19 pandemic.

Methods: We utilized the study of Narrative, a Medical Humanities discipline, as an analytical tool. First, we compiled narratives detailing disagreements between various clinicians and their respective employers' COVID-19 response. Second, we employed literary analysis question prompts to explore the ethical implications of, and potential solutions to, such conflicts.

Results: Physicians, like all individuals, have multiple roles within society. In today's healthcare landscape, many physicians concurrently assume the roles of caregiver and employee. The moral priorities, values, and obligations inherent to these two roles can sometimes be misaligned, creating ethical dilemmas for physicians and their employers.

Conclusion: Physicians must recognize that their primary professional responsibility is to patients. When the welfare or safety of patients appears to be at risk, physicians have a duty to advocate on their behalf. However, physicians and hospital administration alike must seek out synergies and minimize potential conflict. Good hospitals should have forums, communication lines, and organizational cultures that allow clinicians to openly voice concerns and feedback. Good physicians should voice dissenting opinions in a diplomatic, stepwise, and cooperative manner. If patient welfare remains at risk, other recourses are available, but teamwork and collaboration should be the initial focus.

\section{Impact and Implications:}

This discussion can help physicians think deeply about their different roles and responsibilities in society and how to ethically balance those roles. It can also inform various topics within healthcare ethics, including care transformation, resource allocation, organizational ethics, and patient advocacy. 